\title{
An experimental study of a mnemonic system
}

\author{
R. J. SENTER AND GLENN K. HAUSER ${ }^{2}$ \\ UNIVERSITY OF CINCINNATI
}

The learning of a 20 item $\mathrm{CVC}$ list by serial anticipation of Ss receiving a $30 \mathrm{~min}$ training session in a commercial mnemonic technique known as the "hook" or "peg" system was compared with the performance of untrained Ss. Trained Ss performed significantly better, with respect to number of correct anticipations, than did untrained Ss receiving the same trigrams. Trained $S_{s}$ who learned high (100\%) association trigrams exhibited a distortion of the classical serial position effect.

Many authors have exploited a mnemonic technique called the hook or peg system (e.g., Furst, 1949; Furst, 1957; Roth, 1961; Lorayne, 1957). Basically this system involves the overlearning of an ordered series of verbal pegs, usually common English nouns. A new series of words can then be remembered, in the order given, by forming bizarre images associating the new word with the previously memorized pegs. If the peg words are thoroughly overlearned and if the associative images are sufficiently well formed, any word in the newly learned list, along with its position in the list, can be recalled at will (Senter, 1965). The process seems to be an example of "voluntary redintegration" through which the whole memory complex (namely, peg word, list position, and new word) can be recalled by remembering any single element of the complex (usually the peg word).

It is probably because of the aura of commercialism and show business associated with these mnemonic "tricks" that little controlled research investigating the nature and general application of mnemonic systems has been undertaken. The few studies which have been reported are contradictory, with Balaban (1910) and Wallace, Turner, \& Perkins (1957) reporting positive results and Smith \& Noble (1965) reporting generally negative results.

Strong evidence supporting the assertion than mnemonic pegs can act as memory mediators has recently been offered by Wood (1967). Wood, working under the correct assumption that a strongly overlearned list of peg words is as readily available to Ss' consciousness as would be a printed list of those pegs, provided his Ss with a printed list of pegs to be used as mediational tools in the learning of another list of common nouns. Wood's results, under a variety of conditions in five different experiments, indicate that $\mathrm{Ss}$ who used the mnemonic pegs consistently performed at a level significantly superior to Ss who had no access to the mnemonic pegs.

The present study is also an experimental examination of the peg system but uses a method requiring Ss to remember their mnemonic pegs rather than having visual access to them.

\section{Method}

Fifty-two Ss were selected from introductory psychology courses at the University of Cincinnati. After random assignment the $E$ group contained 15 male and 11 female Ss; 13 Ss of each sex comprised the C group.

E group Ss were subjected to a training session (approximately $30 \mathrm{~min}$ ) which consisted of three parts, namely: (1) a demonstration during which $\mathrm{E}$ memorized and recited a list of 20 common nouns created by the Ss; (2) an explanation of the hook or peg mnemonic system (Furst, 1949, pp. 61-81; Lorayne, 1957, pp. 48-59); and (3) an attempt on the part of the Ss to learn a set of 20 nouns using the mnemonic system. $E$ group Ss were required to return one week later and told to practice the mnemonic system in the interim. The Ss were required to participate in research for course credit and were told that they could not be credited with experimental participation unless they had memorized the 20 peg words and practiced using them by the next session.

At the second experimental session (one week later) $\mathrm{E}$ group Ss were given a brief recall test to measure the retention of their 20 peg words. Ss were then required to learn a list of $20 \mathrm{CVC}$ trigrams by the method of serial anticipation with instructions to use their mnemonic system. The CVC list contained ten $0 \%$ association value trigrams alternated with ten $100 \%$ association value trigrams (Hilgard, 1951). The list was shown 10 times by slide projector in a group session with 12 sec exposure and 12 sec intertrial interval. Data were collected during the last nine trials. The Ss' anticipations were written in booklets through a movable mask which covered preceding and succeeding answer spaces.

C group Ss had only one experimental session during which they received a short lecture on memory and were then asked to memorize the CVC list but with no special learning instructions. C group Ss' participation was also required for course credit.

\section{Results}

Figure 1 shows the acquisition curves for both groups and both CVC association levels. The four learning curves indicate quite consistent relationships with the $\mathbf{E}$ group Ss showing performance supertor to their untrained counterparts for the same trigrams. The curve for trained Ss leaming $0 \%$ trigrams is almost superimposed on the curve describing untrained Ss' performance with $100 \%$ trigrams.

Figure 2 shows the total number of correct anticipations for the trigrams in each serial position. The ordinal positions of the groups and stimulus conditions remain the same as was seen in Fig. 1. 


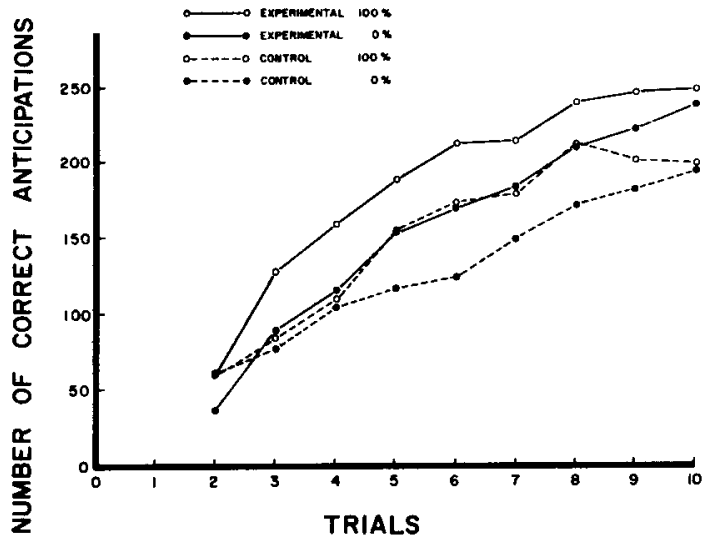

Fig. 1. Total number of trigrams correctly anticipated, as a function of trials, by trained and untrained Ss with $100 \%$ and $0 \%$ association trigrams.

A serial position effect is apparent in its classic form, except for the E-100\% condition where performance on trigrams 10,12 , and 14 is higher than would be expected. Wood (1967) reports Ss using pegs show little or no serial position effect.

The data were subjected to a Treatment (Training) by Level (Per cent Association) by List Position analysis of variance. All three main effects produced significant $F$ ratios (Treatments $-F=8.73$, $d f=1 / 50$, $\mathrm{p}=.01$; Level-F $=68.31, \mathrm{df}=1 / 50, \mathrm{p}=.01$; and List Position $-F=26.41, \mathrm{df}=9 / 450, p=.01$ ). The Treatment by Position and Per cent Association by Position interactions produced significant $F$ ratios of 2.65 and $6.48(\mathrm{df}=9 / 450, p=.01)$, respectively. The Treatments by Per cent Association and the second order interaction were not significant $(p=.05)$.

Discussion

The significant $F$ ratios associated with Per cent Association and Position appear to be classical effects of 'meaningfulness' and serial position. Both significant interactions involve Position effects and are likely the result of the atypically high number of correct anticipations for E-100\% on items 10 , 12 , and 14 .

From Figs. 1 and 2 and the significant Treatments $F$ it seems clear that the performance of Ss receiving mnemonic training was consistently superior to that of control Ss on trigrams of the same association value.

A possible criticism of this study lies in the fact that $\mathbf{E}$ group Ss had a pretesting practice session involving memory practice; the $\mathrm{C}$ group had no equivalent practice. It is possible that nonspecific transfer effects might have contributed to the $E$ group's subsequent performance. It is difficult, however, to believe that only one exposure to dissimilar verbal material (nouns, not trigrams) given under dissimilar conditions (verbally, not by slide projector) a week earlier could have produced transfer

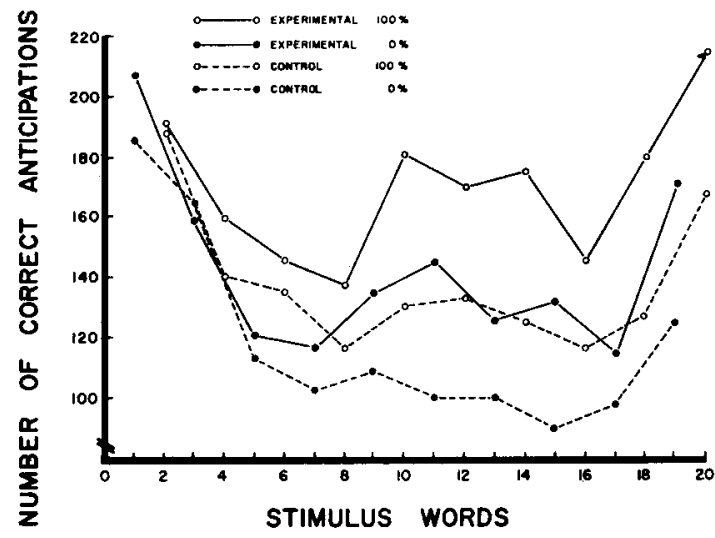

Fig. 2. Frequency of correct anticipation of each trigram as a function of list position. The serial position effect assumes its classic form for all groups except E-100\%.

of sufficient magnitude to raise the $\mathrm{E}$ group's performance on $0 \%$ trigrams to a level virtually equivalent to that shown by the $\mathrm{C}$ group receiving $100 \%$ association trigrams.

It appears that a superficial exposure to the mechanics of the peg system can produce measurable and statistically reliable differences in Ss' performance on a standard laboratory learning task. This study is interpreted as adding confirmation to the results of Balaban (1910), Wallace et al (1957), and Wood (1967). Evidence is lent to the hypothesis that voluntary redintegration, as exemplified by the peg system, can enhance the process of rote memorizing.

\section{References}

BALABAN, A. Uber den Unterschied des logischen und mechanischen Gedächtnisses. Z. Psychol, 1910, 56, 356-377.

FURST, B. Stop Forgetting. New York: Greenburg, 1949.

FURST, B. The practical way to a better memory. New York: Fawcett World Library, 1957.

HILGARD, E. R. Methods and procedures in the study of learning. In S. S. Stevens (Ed.), Handbook of experimental psychology. New York: Wiley, 1951, 517-568.

LORAYNE, H. How to develop a super-power memory. New York: F. Fell, 1957.

ROTH, D. M. Roth memory course. Santa Monica: Motivation, 1961. SENTER, R. J. Review of mnemonics and mnemonotechnics for improved memory. Wright-Patterson Air Force Base, Ohio: Aerospace Medical Research Laboratories, 1965 (AMRL-TR-65-180). SMITH, R., \& NOBLE, C. E. Effects of a mnemonic technique applied to verbal learning and memory. Percept. mot. Skills, 1965, 21, 123-134.

WALLACE, W. H., TURNER, S. H., \& PERKINS, C. C. Preliminary studies of human information storage. Signal Corps Project No. 1320, Institute for Cooperative Research, University of Pennsylvania, 1957. WOOD, G. Mnemonic systems in recall. J. educ. Psychol. Monogr., $1967,58$.

\section{Notes}

1. This research was submitted by the junior author in partial fulfillment of his requirements for the B.A. at the University of Cincinnati. 2. Now at Lehigh University. 